\title{
Some Chaotic Properties of a Kind of Coupled Map Lattices
}

\author{
Xiaofang Yang', Tianxiu Lu ${ }^{1,2 *}$, Guo Liu ${ }^{1}$ \\ ${ }^{1}$ Department of Mathematics and Statistics, Sichuan University of Science and Engineering, Zigong, China \\ ${ }^{2}$ Artificial Intelligence Key Laboratory of Sichuan Province, Zigong, China \\ Email: xf_suse@163.com, *lubeeltx@163.com,lg0506@outlook.com
}

How to cite this paper: Yang, X.F., Lu, T.X. and Liu, G. (2020) Some Chaotic Properties of a Kind of Coupled Map Lattices. Journal of Applied Mathematics and Physics, 8, 968-975.

https://doi.org/10.4236/jamp.2020.86075

Received: May 7, 2020

Accepted: May 28, 2020

Published: June 1, 2020

Copyright $\odot 2020$ by author(s) and Scientific Research Publishing Inc. This work is licensed under the Creative Commons Attribution International License (CC BY 4.0).

http://creativecommons.org/licenses/by/4.0/

\begin{abstract}
This paper is concerned with some chaotic properties of a kind of coupled map lattices, which is proposed by Kaneko. First, this research discussed the sensitivity, infinite sensitivity, transitivity, accessibility, densely Li-Yorke sensitivity and exact of coupled map lattices. Then, some sufficient conditions under which $\left(\Delta_{\infty}^{\infty}, d,\left.F\right|_{\Delta_{\infty}^{\infty}}\right)$ is Kato chaotic, positive entropy chaotic and Ruelle-Takens chaos are obtained.
\end{abstract}

\section{Keywords}

Coupled Map Lattices, Sensitivity, Chaos, Accessibility

\section{Introduction}

In 1983, Kaneko [1] proposed coupled map lattices (Short for CMLs). Then, in biophysics, materials, chaos, image processing, CMLs are intensively discussed (Refer to literature [2]-[8] and others). In 2005, the literature [9] showed that CMLs have some topology and ergodic properties. In 2010, Juan Lu [10] presented a definition of distributional chaos on a sequence (DCS) for CML systems and stated two different sufficient conditions for having DCS. In 2010, Juan Luis [11] proved that this CML system has positive topological entropy for zero coupling constant. In 2016, Risong Li [12] [13] had obtained some relevant conclusions for the zero coupling constant and proved that the system has three kinds of chaos. In this paper, the following CML from [14] is considered.

$$
x_{m+1, n}=(1-\varepsilon) f\left(x_{m, n}\right)+\varepsilon f\left(x_{m, n-1}\right)
$$

where $\quad x_{m, n} \in I, \quad m \in \mathbb{N}_{0}=\{0,1,2, \cdots\}, \quad n \in \mathbb{Z}=\{\cdots,-1,0,1, \cdots\}, \quad I$ is a non-degenerate compact interval, $f$ is a map on $I$, and $\varepsilon \in[0,1]$ is a constant. 
For $t \in \mathbb{Z}$, let $\mathbb{N}_{t}=\{t, t+1, \cdots\}$ and $\Omega=\{(0, n): n \in \mathbb{Z}\}=\{\cdots,(0,-1),(0,0),(0,1), \cdots\}$. For any sequence $\phi=\left\{\phi_{0, n}\right\}_{\infty}^{\infty}$ on $\Omega$, by induction, one can obtain a double-indexed sequence $x=\left\{x_{m, n}: m=0,1,2, \cdots ; n=\cdots,-1,0,1, \cdots\right\}$, which is said to be a solution of the above system (1) with initial condition $\phi$.

Let $I$ be a subset of real number set, write

$$
I_{\infty}^{\infty}=\left\{\left\{a_{n}\right\}_{n=-\infty}^{\infty}=\left(\cdots, a_{-1}, a_{0}, a_{1}, \cdots\right): a_{n} \in I, n \in \mathbb{Z}\right\}
$$

and

$$
\Delta_{\infty}^{\infty}=\left\{\left(\cdots, a_{-1}, a_{0}, a_{1}, \cdots\right): a_{i}=a_{j} \in I, i, j \in \mathbb{Z}\right\}
$$

which is called the diagonal set of $I_{\infty}^{\infty}$.

For arbitrary, two sequences $x_{1}=\left\{x_{1, n}\right\}_{n=-\infty}^{\infty}, \quad x_{2}=\left\{x_{2, n}\right\}_{n=-\infty}^{\infty} \in I_{\infty}^{\infty}$, it is easy to prove that

$$
d\left(x_{1}, x_{2}\right)=\sup \left\{\left|x_{1, n}-x_{2, n}\right|: n=\cdots,-1,0,1, \cdots\right\}
$$

is a metric on $I_{\infty}^{\infty}$.

Let $f: I \mapsto I$ be a continuous map and $x=\left\{x_{m, n}: m \in \mathbb{N}_{0}, n \in \mathbb{Z}\right\}$ be a solution of the above system (1) with initial condition $\phi=\left\{\phi_{0, n}\right\}_{\infty}^{\infty} \in I_{\infty}^{\infty}$.

Let

$$
x_{m}=\left\{x_{m, n}\right\}_{n=-\infty}^{\infty}=\left(\cdots, x_{m,-1}, x_{m, 0}, x_{m, 1}, \cdots\right), \forall m \in \mathbb{N}_{0},
$$

and

$$
x_{m+1}=\left\{x_{m+1, n}\right\}_{n=-\infty}^{\infty}=\left(\cdots, x_{m+1,-1}, x_{m+1,0}, x_{m+1,1}, \cdots\right)=F\left(x_{m}\right), \forall m \in \mathbb{N}_{0},
$$

where $x_{0}=\phi=\left\{x_{0, n}=\phi_{0, n}\right\}_{n=-\infty}^{\infty}$ and

$$
x_{m+1, n}=(1-\varepsilon) f\left(x_{m, n}\right)+\varepsilon f\left(x_{m, n-1}\right), \forall m \in \mathbb{N}_{0}, n \in \mathbb{Z} .
$$

Then, one can see that the above system (1) is equivalent to the following system

$$
x_{m+1}=F\left(x_{m}\right), x_{m} \in I_{\infty}^{\infty}, m=0,1,2, \cdots
$$

For the above system (3), the map $F$ is said to be induced by the system (1). Obviously, a double-indexed sequence $\left\{x_{m, n}: m \in \mathbb{N}_{0}, n \in \mathbb{Z}\right\}$ is a solution of the above system (1) if and only if the sequence $\left\{x_{m}=\left\{x_{m, n}\right\}_{n=-\infty}^{\infty}: m \in \mathbb{N}_{0}\right\}_{m=0}^{\infty}$ is a solution of the above system (3).

Next section, the definitions of sensitive, infinite sensitive, transitive, accessibility, densely Li-Yorke sensitive and exact will be reviewed. And then, in section 3 , it is proved that the system $\left(\Delta_{\infty}^{\infty}, d,\left.F\right|_{\Delta_{\infty}^{\infty}}\right)$ satisfies three definitions of chaos (Kato chaotic, positive entropy chaotic and Ruelle-Takens chaos) under the conditions that $f$ is chaos in these sense.

\section{Preliminaries}

After T. Y. Li and J. A. Yorke [15] first put forward the mathematical definition of "chaos", many other definitions of chaos appeared later. For example, sensi- 
tivity, infinite sensitivity, transitivity, accessibility, densely Li-Yorke sensitivity, Kato chaotic, positive entropy chaotic, Ruelle-Takens chaos, and so on.

Definition 1. Let $(X, \rho)$ be a metric space and $f: X \mapsto X$ be a continuous function. $f$ is said to be

1) transitive if for any nonempty open subsets $U_{1}, U_{2} \subset Y, f^{n}\left(U_{1}\right) \cap U_{2} \neq \varnothing$ for some integer $n \in \mathbb{N}$ (see [16]).

2) sensitive if there exist $\eta>0$ such that for any $x \in X$ and $\varepsilon>0$, there exists $y \in B(x, \varepsilon)$ and $n \in \mathbb{N}$ such that $\rho\left(f^{n}(x), f^{n}(y)\right)$ (see [17]).

3) infinitely sensitive if there exist $\eta>0$ such that for any $x \in X$ and $\varepsilon>0$, there exists $y \in B(x, \varepsilon)$ and $n \in \mathbb{N}$ such that $\limsup \rho\left(f^{n}(x), f^{n}(y)\right) \geq \eta \quad($ see [17]).

$\left.{ }_{4}^{n}\right)^{\infty}$ accessible if for any $\varepsilon>0$ and any two nonempty open subsets $U_{1}, U_{2} \subset X$, there are two points $x \in U_{1}$ and $y \in U_{2}$ such that $\rho\left(f^{n}(x), f^{n}(y)\right)<\varepsilon$ for some integer $n>0$ (see [16]).

5) exact if for any open subset $U \subset X$, there is $m \in \mathbb{N}$ such that $f^{m}(U)=X \quad($ see $[18])$.

Remark 1. [19] There is another equivalent definition of transitivity. $f: X \mapsto X$ is said to be transitivity, if there is an $x_{0} \in X$ such that $\overline{\operatorname{Orb}_{f}\left(x_{0}\right)}=X$. Where, $\overline{\operatorname{Orb}_{f}\left(x_{0}\right)}=\left\{f^{n}\left(x_{0}\right) \mid n=0,1,2, \cdots\right\}$ is called the orbit of the point $x_{0}$.

Definition 2. 1) A dynamic system $(X, f)$ (or the map $f: X \rightarrow X$ ) is Li-Yorke sensitive, if for any $x \in X$ has $x \in Q_{\delta}(f)$ for some $\delta>0$.

2) A dynamic system $(X, f)$ (or the map $f: X \rightarrow X$ ) is densely Li-Yorke sensitive if $Q_{\delta}(f)$ is dense in $X$ for some $\delta>0$. Among them,

$$
\begin{gathered}
Q_{\delta}(f)=\left\{x \in X: \forall \varepsilon>0, \exists y \in B(x, \varepsilon) \text { such that }(x, y) \in L Y_{\rho}(f, \delta)\right\} \\
L Y_{\rho}(f, \delta)=\left\{(x, y) \in X \times X: \limsup _{n \rightarrow \infty} \rho\left(f^{n}(x), f^{n}(y)\right)>\delta\right. \\
\text { and } \left.\liminf _{n \rightarrow \infty} \rho\left(f^{n}(x), f^{n}(y)\right)=0\right\}
\end{gathered}
$$

Definition 3. 1) A dynamic system $(X, f)$ (or the map $f: X \rightarrow X)$ is Kato chaotic if it is sensitive and accessible (see [20]).

2) A dynamic system $(X, f)$ (or the map $f: X \rightarrow X)$ is chaotic in the sense of Ruelle and Takens (short for $R$-T chaotic) if it is transitive and sensitive (see [21]).

Proposition 1. A dynamic system $(X, f)$ (or the map $f: X \rightarrow X$ ) is Li-Yorke sensitive if and only if $\overline{P_{\delta}(f)}=X$ for some $\delta>0$. Among them,

$$
P_{\delta}(f)=\left\{x \in X: \forall \varepsilon>0, \exists y \in B(x, \varepsilon), \exists n \in \mathbb{N} \text { such that } \rho\left(f^{n}(x), f^{n}(y)\right)>\delta\right\}
$$

Proposition 2. [17] $A$ dynamical system $(X, f)$ is infinitely sensitive if and only is it is sensitive.

Proposition 3. [22] A dynamical system $(X, f)$ is dense Li-Yorke sensitivity, then it is Topological mixing (or its topological entropy is positive). 


\section{Main Results}

In this section, let $X=I$. The metric $\rho$ in $I$ is defined by $\rho(a, b)=|a-b|(\forall a, b \in I)$. The metric $d$ in $I_{\infty}^{\infty}$ is defined by (2).

Theorem 1. If $\mathrm{f}$ is transitive, then the system $\left(\Delta_{\infty}^{\infty}, d,\left.F\right|_{\Delta_{\infty}^{\infty}}\right)$ is transitive.

Proof. Since $f$ is transitive, then there exist $a \in I$ satisfying $\overline{\operatorname{Orb}_{f}(a)}=I$. Then for any $b \in I$ and any $\varepsilon>0, B(b, \varepsilon) \cap \operatorname{Orb}_{f}(a) \neq \varnothing$. That is, there exists a $k_{0}>0$ such that $\rho\left(f^{k_{0}}(a), b\right)=\left|f^{k_{0}}(a)-b\right|<\varepsilon$. Take $x_{0}=(\cdots, a, a, a, \cdots) \in \Delta_{\infty}^{\infty}$. It is easy to see, for any $k \in \mathbb{N}$, $F^{k}\left(x_{0}\right)=\left\{f^{k}(a)\right\}_{n=-\infty}^{\infty}$. Then, $\operatorname{Orb}_{F}\left(x_{0}\right)=\left.\left\{f^{k}(a)\right\}_{n=-\infty}^{\infty}\right|_{k \in \mathbb{N}}$. For any $y=(\cdots, b, b, b, \cdots) \in \Delta_{\infty}^{\infty}$ and above $k_{0}>0$,

$$
d\left(F^{k_{0}}\left(x_{0}\right), y\right)=\sup \left\{\left|f^{k_{0}}(a)-b\right|: n \in \mathbb{N}\right\}=\left|f^{k_{0}}(a)-b\right|<\varepsilon .
$$

So, $B(y, \varepsilon) \cap \operatorname{Orb}_{F}\left(x_{0}\right) \neq \varnothing$.

Thus, the system $\left(\Delta_{\infty}^{\infty}, d,\left.F\right|_{\Delta_{\infty}^{\infty}}\right)$ is transitive.

Theorem 2. If $\mathrm{f}$ is sensitive, then the system $\left(\Delta_{\infty}^{\infty}, d,\left.F\right|_{\Delta_{\infty}^{\infty}}\right)$ is sensitive.

Proof. Take $\Delta_{\infty}^{\infty}=\left\{\left(\cdots, x_{-1}, x_{0}, x_{0}, \cdots\right): x_{n}=a \in I, n \in \mathbb{Z}\right\} \subset I_{\infty}^{\infty}$,

$\forall x=\{\cdots, a, a, a, \cdots\}, \quad y=\{\cdots, b, b, b, \cdots\} \in \Delta_{\infty}^{\infty}, \quad x \neq y$. It is easy to know that, for $\forall k \in \mathbb{N}$,

$$
F^{k}(x)=\left\{f^{k}(a)\right\}_{n=-\infty}^{\infty}, F^{k}(y)=\left\{f^{k}(b)\right\}_{n=-\infty}^{\infty}
$$

So, for $\forall k \in \mathbb{N}$,

$$
\begin{aligned}
d\left(F^{k}(x), F^{k}(y)\right) & =d\left(\left\{f^{k}(a)\right\}_{n=-\infty}^{\infty},\left\{f^{k}(b)\right\}_{n=-\infty}^{\infty}\right) \\
& =\sup \left\{\left|f^{k}(a)-f^{k}(b)\right|, k=\cdots,-1,0,1, \cdots\right\} \\
& =\left|f^{k}(a)-f^{k}(b)\right| .
\end{aligned}
$$

Since $f$ is Sensitive, so there exists a $\varepsilon_{0}>0$ such that for any $p \in I$ and any $\delta>0$, there exists a $q_{p, \delta} \in B(p, \delta)$ and $n_{p, \delta} \in \mathbb{N}$ such that $\left|f^{n_{p, \delta}}(p), f^{n_{p, \delta}}\left(q_{p, \delta}\right)\right|>\varepsilon_{0}$. So for any fixed $x=(\cdots, p, p, p, \cdots) \in \Delta_{\infty}^{\infty}$ and any $\delta>0$, taking $y=\left(\cdots, q_{p, \delta}, q_{p, \delta}, q_{p, \delta}, \cdots\right) \in \Delta_{\infty}^{\infty}$, one has that,

$$
d(x, y)=\sup \left\{\cdots,\left|p-q_{p, \delta}\right|,\left|p-q_{p, \delta}\right|,\left|p-q_{p, \delta}\right|, \cdots\right\}=\left|p-q_{p, \delta}\right|<\delta,
$$

that is $y \in B(x, \delta)$. And because

$$
d\left(F^{n_{p, \delta}}(x), F^{n_{p, \delta}}(y)\right)=\left|f^{n_{p, \delta}}(p)-f^{n_{p, \delta}}\left(q_{p, \delta}\right)\right|>\varepsilon_{0},
$$

so $\left.F\right|_{\Delta_{\infty}^{\infty}}$ is sensitive.

Corollary 1. If $f$ is chaotic in the sense of Ruelle and Takens, then the system $\left(\Delta_{\infty}^{\infty}, d,\left.F\right|_{\Delta_{\infty}^{\infty}}\right)$ is chaotic in the sense of Ruelle and Takens.

Proof. According to Theorem 1, Theorem 2 and the definition of R-T chaos, 
the conclusion is obvious.

According to Proposition 2 and Theorem 2, the following Corollary is hold.

Corollary 2. If $f$ is infinitely sensitive, then the system $\left(\Delta_{\infty}^{\infty}, d,\left.F\right|_{\Delta_{\infty}^{\infty}}\right)$ is sensitive.

In fact, there is a stronger conclusion.

Theorem 3. If $f$ is infinitely sensitive, then the system $\left(\Delta_{\infty}^{\infty}, d,\left.F\right|_{\Delta_{\infty}^{\infty}}\right)$ is infinitely sensitive.

Proof. Since $f$ is infinitely sensitive, then there exists a $\delta>0$ such that for any $a \in I$ and any $\varepsilon$, there exists $b_{a, \varepsilon} \in B(a, \varepsilon)$ and $n_{a, \varepsilon} \in \mathbb{N}$ such that $\limsup \rho\left(f^{n_{a, \varepsilon}}(a), f^{n_{a, \varepsilon}}\left(b_{a, \varepsilon}\right)\right) \geq \delta$. So for any fixed $x=(\cdots, a, a, a, \cdots) \in \Delta_{\infty}^{\infty}$, and any $\varepsilon>0$, taking $x=\left(\cdots, b_{a, \varepsilon}, b_{a, \varepsilon}, b_{a, \varepsilon}, \cdots\right) \in \Delta_{\infty}^{\infty}$, one has that

$$
d(x, y)=\sup \left\{\cdots,\left|a-b_{a, \varepsilon}\right|,\left|a-b_{a, \varepsilon}\right|,\left|a-b_{a, \varepsilon}\right|, \cdots\right\}=\left|a-b_{a, \varepsilon}\right|<\varepsilon,
$$

that is $y \in B(x, \varepsilon)$. And because

$$
\limsup _{n_{a, \varepsilon}} d\left(F^{n_{a, \varepsilon}}(x), F^{n_{a, \varepsilon}}(y)\right)=\limsup _{n_{a, \varepsilon} \rightarrow \infty} d\left(f^{n_{a, \varepsilon}}(a), f^{n_{a, \varepsilon}}\left(b_{a, \varepsilon}\right)\right) \geq \delta .
$$

So $\left.F\right|_{\Delta_{\infty}^{\infty}}$ is infinitely sensitive.

Theorem 4. If $\mathrm{f}$ is accessible, then the system $\left(\Delta_{\infty}^{\infty}, d,\left.F\right|_{\Delta_{\infty}^{\infty}}\right)$ is accessible.

Proof. For any open subset

$$
\left(\Delta_{1}\right)_{\infty}^{\infty}=\left\{\left(\cdots, x_{-1}, x_{0}, x_{1}, \cdots\right), x_{n}=a \in U_{1} \subset I, n \in \mathbb{Z}\right\} \subset I_{\infty}^{\infty}
$$

and

$$
\left(\Delta_{2}\right)_{\infty}^{\infty}=\left\{\left(\cdots, y_{-1}, y_{0}, y_{1}, \cdots\right), y_{n}=b \in U_{2} \subset I, n \in \mathbb{Z}\right\} \subset I_{\infty}^{\infty},
$$

since $f$ is accessible, then, for the above $U_{1}, U_{2} \subset I$, there exist $a \in U_{1}, b \in U_{2}$ such that

$$
\rho\left(f^{k}(a), f^{k}(b)\right)=\left|f^{k}(a)-f^{k}(b)\right|<\varepsilon
$$

for some $k>0$. Take

$$
x=(\cdots, a, a, a, \cdots) \in\left(\Delta_{1}\right)_{\infty}^{\infty}, y=(\cdots, b, b, b, \cdots) \in\left(\Delta_{2}\right)_{\infty}^{\infty},
$$

then

$$
d\left(F^{k}(x), F^{k}(y)\right)=\left|f^{k}(a), f^{k}(b)\right|<\varepsilon .
$$

So, the system $\left(\Delta_{\infty}^{\infty}, d,\left.F\right|_{\Delta_{\infty}^{\infty}}\right)$ is accessible.

Corollary 3. If $\mathrm{f}$ is Kato chaotic, then the system $\left(\Delta_{\infty}^{\infty}, d,\left.F\right|_{\Delta_{\infty}^{\infty}}\right)$ is Kato chaotic.

Proof. According to Theorem 2 and Theorem 4, the conclusion is obvious.

Theorem 5. If $\mathrm{fis}$ exact, then the system $\left(\Delta_{\infty}^{\infty}, d,\left.F\right|_{\Delta_{\infty}^{\infty}}\right)$ is exact.

Proof. Since $f$ is exact, for any open subset $D \subset I$, there exist $m \in \mathbb{N}$ such that $f^{m}(D)=I$. That is, for any $a \in D$, there exists an $m>0$ such that $B\left(f^{m}(a), \varepsilon\right) \cap I \neq \varnothing$ for any $\varepsilon>0$. So there is a $b \in X$ such that $\rho\left(f^{m}(a), \varepsilon\right)=\left|f^{m}(a)-b\right|<\varepsilon$. 
Take $\left(\Delta^{*}\right)_{\infty}^{\infty}$ is arbitrary open subset of $\Delta_{\infty}^{\infty}$, and $x_{0}=(\cdots, a, a, a, \cdots) \in\left(\Delta^{*}\right)_{\infty}^{\infty}$. Clearly, for any $k \in \mathbb{N}, F^{k}\left(x_{0}\right)=\left\{f^{k}(a)\right\}_{n=-\infty}^{\infty}$. For any $y_{0}=(\cdots, b, b, b, \cdots) \in \Delta_{\infty}^{\infty}, d\left(F^{m}\left(x_{0}\right), y_{0}\right)=\left|f^{m}(a)-b\right|<\varepsilon$. That is to say, there exist an $m \in \mathbb{N}, F^{m}\left(\left(\Delta^{*}\right)_{\infty}^{\infty}\right)=\Delta_{\infty}^{\infty}$. So, the system $\left(\Delta_{\infty}^{\infty}, d,\left.F\right|_{\Delta_{\infty}^{\infty}}\right)$ is exact.

In [23] we had proved that, $f$ is Li-Yorke sensitive implies that the system $\left(\Delta_{\infty}^{\infty}, d,\left.F\right|_{\Delta_{\infty}^{\infty}}\right)$ is Li-Yorke sensitive. Inspired by this, the following conclusion can be drawing.

Theorem 6. If $\mathrm{f}$ is densely Li-Yorke sensitive, then the system $\left(\Delta_{\infty}^{\infty}, d,\left.F\right|_{\Delta_{\infty}^{\infty}}\right)$ is densely Li-Yorke sensitive.

Proof. Since $f$ is densely Li-Yorke sensitive, then for any $a \in Q_{\delta}(f)$ and any $\varepsilon>0$. Then there exists a $b \in B(a, \varepsilon)$ such that $(a, b) \in L Y_{\rho}(f, \delta)$. Take $x^{*}=\left\{x_{n}=a\right\}_{n=-\infty}^{\infty}, \quad y^{*}=\left\{y_{n}=b\right\}_{n=-\infty}^{\infty}$. One has that

$$
\limsup _{n \rightarrow \infty} d\left(F^{n}\left(x^{*}\right), F^{n}\left(y^{*}\right)\right)=\underset{n \rightarrow \infty}{\limsup } d\left(f^{n}(a), f^{n}(b)\right)>\delta
$$

and

$$
\liminf _{n \rightarrow \infty} d\left(F^{n}\left(x^{*}\right), F^{n}\left(y^{*}\right)\right)=\liminf _{n \rightarrow \infty} d\left(f^{n}(a), f^{n}(b)\right)=0
$$

Thus there is an $x^{*} \in Q_{\delta}(F)$.

Any fixed $x \in \Delta_{\infty}^{\infty}$, write $x=\left(\cdots, x_{m,-1}, x_{m, 0}, x_{m, 1}, \cdots\right)$, where $x_{m, p}=x_{m, p+1}, p \in \mathbb{Z}$. Because $f: I \mapsto I$ is densely Li-Yorke sensitive, then for any $\varepsilon>0$ and the above $x_{m, 0}, B\left(x_{m, 0}, \varepsilon\right) \cap Q_{\delta}(f) \neq \varnothing$. Take $a \in B\left(x_{m, 0}, \varepsilon\right) \cap Q_{\delta}(f)$, then

$$
d\left(x, x^{*}\right)=\sup \left\{\left|x_{m, p}-a\right|\right\}=\left|x_{m, p}-a\right|<\varepsilon .
$$

So $x^{*} \in B(x, \varepsilon)$. This suggests that $\overline{Q_{\delta}(F)}=\Delta_{\infty}^{\infty}$.

So, the system $\left(\Delta_{\infty}^{\infty}, d,\left.F\right|_{\Delta_{\infty}^{\infty}}\right)$ is densely Li-Yorke sensitive.

According to Proposition 3 and Theorem 6 the following is right.

Corollary 4. If $f$ is dense Li-Yorke sensitivity, then the system $\left(\Delta_{\infty}^{\infty}, d,\left.F\right|_{\Delta_{\infty}^{\infty}}\right)$ it is Topological mixing (or its topological entropy is positive).

\section{Conclusion}

Inspired by the literature [23], this paper further studies the chaoticity of coupled map lattices. Some sufficient conditions of sensitivity, accessibility and transitivity are obtained. However, the study of coupled map lattices is still a hot topic. Based on the conclusions of this paper and others, one can consider some questions, such as the form of CMLs, the measurement of CMLs, and discuss the chaos of CMLs in other systems, which are worthy of studying.

\section{Conflicts of Interest}

The authors declare no conflicts of interest regarding the publication of this paper. 


\section{References}

[1] Kaneko, K. (1983) Theory and Application of Coupled Map Lattices. John Wiley and Sons, Ann Arbor.

[2] Chen, T., Chen, Y. and Gong, M. (2019) A Coupled-Channel Lattice Study of the Resonance-Like Structure $Z_{c}(3900)$. Chinese Physics C, 43, 103-103. https://doi.org/10.1088/1674-1137/43/10/103103

[3] Proscia N., Collison R. J., Meriles C.A. and Menon, V.M. (2019) Coupling of Deterministically Activated Quantum Emitters in Hexagonal Boron Nitride to Plasmonic Surface Lattice Resonances. Nanophotonics, 8, 876-894. https://doi.org/10.1515/nanoph-2019-0136

[4] Syga, S., Nava-Sedeño, J.M., Brusch, L. and Deutsch, A. (2019) A Lattice-Gas Cellular Automaton Model for Discrete Excitable Media. Spirals and Vortices, 5, 253-264. https://doi.org/10.1007/978-3-030-05798-5 15

[5] Chaté, H. and Manneville, P. (1988) Spatio-Temporal Intermittency in Coupled Map Lattices. Physica D: Nonlinear Phenomena, 32, 409-422.

https://doi.org/10.1016/0167-2789(88)90065-6

[6] Guirao, J.L.G. and Lampart, M. (2010) Positive Entropy of a Coupled Lattice System Related with Belusov-Zhabotinskii Reaction. Journal of Mathematical Chemistry, 48, 66-71. https://doi.org/10.1007/s10910-009-9624-3

[7] Kawaguchi, M., Shimomoto, K., Shibata, A. and Kato, T. (1999) Effect of Anisotropy on Viscous Fingering Patterns of Polymer Solutions in Linear Hele-Shaw Cells. Chaos, 9, 323-328. https://doi.org/10.1063/1.166409

[8] Afraimovich, V.S. and Chow, S.-N. (1995) Topological Spatial Chaos and Homoclinic Points of $\mathrm{Z}^{\mathrm{d}}$-Actions in Lattice Dynamical Systems. Japan Journal of Industrial \& Applied Mathematics, 12, Article No. 367. https://doi.org/10.1007/BF03167235

[9] Afraimovich, V., Chazottes, J.-R. and Fernandez, B. (2005) Some Topological Properties of Lattice Dynamical Systems. Dynamics of Coupled Map Lattices \& of Related Spatially Extended Systems, 1, 153-179. https://doi.org/10.1007/11360810 7

[10] Guirao, J.L.G. (2010) Distributional Chaos of Generalized Belusov-Zhabotinskii's Reaction Models. Match Communications in Mathematical \& in Computer Chemistry, 2, 23-33.

[11] Guirao, J.L.G. and Lampart, M. (2010) Positive Entropy of a Coupled Lattice System Related with Belusov-Zhabotinskii Reaction. Journal of Mathematical Chemistry, 48, 66-71.

[12] Li, R.S., Zhao, Y., Jiang, R. and Wang, H.Q. (2016) Some Remarks on Chaos of a Coupled Lattice System Related with the Belusov-Zhabotinskii Reaction. Journal of Mathematical Chemistry, 54, 849-853. https://doi.org/10.1007/s10910-016-0616-9

[13] Li, R., Wang, J., Lu, T.X. and Jiang, R. (2016) Remark on Topological Entropy and $\rho$-Chaos of a Coupled Lattice System with Non-Zero Coupling Constant Related with Belusov-Zhabotinskii Reaction. Journal of Mathematical Chemistry, 54, 1110-1116. https://doi.org/10.1007/s10910-016-0609-8

[14] Lu, T.X. and Li, R.S. (2017) Some Chaotic Properties of a Coupled Lattice System Related with Belusov-Zhabotinsky Reaction. Qualitative Theory of Dynamical Systems, 16, 657-670. https://doi.org/10.1007/s12346-016-0210-3

[15] Li, T.-Y. and Yorke, J.A. (1975) Periodic Three Implies Chaos. The American Mathematical Monthly, 82, 985-992. https://doi.org/10.1080/00029890.1975.11994008

[16] Wang, L.D., Peng, J. and Liu, H. (2010) Chaos Caused by a Transitive Map in Information. E-Business Technology \& Strategy-international Conference, 2, 124-129. 
https://doi.org/10.1007/978-3-642-16397-5 11

[17] Wu, X.X. and Zhu, P.Y. (2012) Dense Chaos and Densely Chaotic Operators. Tsukuba Journal of Mathematics, 36, 367-375.

https://doi.org/10.21099/tkbjm/1358777004

[18] Kwietniak, D. and Misiurewicz M. (2005) Exact Devaney Chaos and Entropy. Qualitative Theory of Dynamical Systems, 6, Article No.: 169. https://doi.org/10.1007/BF02972670

[19] Devaney, R.L. (1988) An Introduction to Chaotic Dynamical Systems. Acta Applicandae Mathematica, 13, 221-226. https://doi.org/10.1007/BF00047506

[20] Kato, H. (1996) Everywhere Chaotic Homeomorphisms on Manifields and $k$ Dimensional Menger Manifolds. Topology and its Applications, 72, 1-17. https://doi.org/10.1016/0166-8641(96)00008-9

[21] Gu, R.B. (2007) Kato's Chaos in Set-Valued Discrete Systems. Chaos, Soliton \& Fractals, 31, 765-771. https://doi.org/10.1016/j.chaos.2005.10.041

[22] $\mathrm{Wu}, \mathrm{X} . \mathrm{X}$. and Zhu, P.Y. (2012) The Principal Measure and Distributional ( $p$, $q$ )-Chaos of a Coupled Lattice System Related with Belusov-Zhabotinskii Reaction. Journal of Mathematical Chemistry, 50, 2439-2445. https://doi.org/10.1007/s10910-012-0041-7

[23] Lu, T.X., Zhu, P.Y. and Wu, X.X. (2013) Some Chaotic Properties of Coupled Map Lattices. Journal of Systems Science and Mathematical Sciences, 9, 1119-1128. 